\title{
Structural Coloring in Large Scale Core-Shell Nanowires
}

\author{
Tural Khudiyev, ${ }^{+, \neq}$Erol Ozgur, ${ }^{+, \neq}$Mecit Yaman, ${ }^{+, \neq}$and Mehmet Bayindir, ${ }^{*,+,, \S}$ \\ ${ }^{\dagger}$ UNAM-National Nanotechnology Research Center, ${ }^{\ddagger}$ Institute of Materials Science and Nanotechnology, and ${ }^{\S}$ Department of Physics, \\ Bilkent University, 06800 Ankara, Turkey
}

Supporting Information

ABSTRACT: We demonstrated two complementary size-dependent structural coloring mechanisms, interference and scattering, in indefinitely long core-shell nanowire arrays. The unusual nanostructures are comprised of an amorphous semiconducting core and a polymer shell layer with disparate refractive indices but with similar thermomechanical properties. Core-shell nanowires are mass produced from a macroscopic semiconductor rod by using a new top-to-bottom fabrication approach based on thermal size reduction. Nanostructures with diameters from 30 to $200 \mathrm{~nm}$ result in coloration that spans the whole visible spectrum via

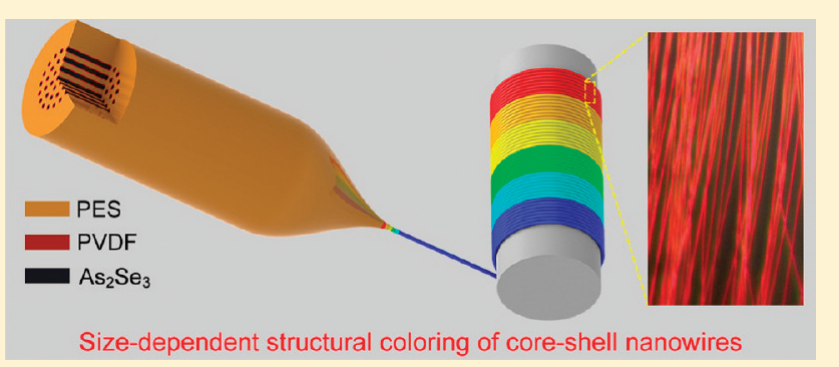
resonant Mie scattering. Nanoshell coloration based on thin film interference is proposed as a structural coloration mechanism which becomes dominant for nanowires having 700-1200 nm diameter. Controlled color generation in any part of visible and infrared spectral regions can be achieved by the simple scaling down procedure. Spectral color generation in mass-produced uniform core-shell nanowire arrays paves the way for applications such as spectral authentication at nanoscale, light-scattering ingredients in paints and cosmetics, large-area devices, and infrared shielding.

KEYWORDS: Top-to-bottom approach, thermal size reduction, core-shell nanowires, thin-film interference, structural coloring, resonant Mie scattering

Tntensely bright structural colors ${ }^{1,2}$ arise not from quantum Loptical interactions as in dye pigments but from the rich phenomena of light propagation in low or nonabsorbing, periodic, or quasi-periodic structures on the length scale of the wavelength of visible light. These phenomena include fundamental light-matter interactions such as thin film, multilayer interference $^{3,4}$ and diffraction grating effects, and also resonant light scattering from small particles, photonic crystals, and plasmonic nanostructures. ${ }^{5}$ In nature, structural coloring can be observed in macroscopic objects with submicrometer and nanosized features. For example, typical bright, iridescent colors observed in insects and birds ${ }^{6}$ result from large scale nanostructures. Therefore, for artificial structural coloring, large scale and high throughput nanostructures must be produced that can be, ideally, employed for both interference and scattering effects. ${ }^{7,8}$

Light-material interactions at the nanoscale are being extensively studied in nanowires and other one-dimensional nanomaterials. For example, color tunability of individual silicon nanowires ${ }^{9}$ and multicolored vertical silicon nanowires ${ }^{10}$ are two recent demonstrations of resonant nanowire and electromagnetic wave interactions. Beside coloration, light confinement, leaky-mode resonances, and nonresonant interference effects can be used to tune and enhance light absorption in a wide range of highperformance, nanowire-based photonic and optoelectronic devices, including lasers, ${ }^{11}$ solar cells, ${ }^{12}$ chemosensors, and photodetectors. ${ }^{13}$ However these examples are exclusively for single dielectric or semiconducting nanowires or their arrays that cannot be easily scaled up for mass production. Here we report unique coloration implications of large scale nanowire and core-shell structures obtained by a novel thermal size reduction technique. ${ }^{14}$ By use of an iterative thermal size reduction method, polymer embedded, indefinitely long, semiconductor/polymer core-shell nanowires are mass-produced. The core-shell nanostructures feature both interference and scattering based coloration. We propose interference based coloration in nanostructures as a new mechanism for large scale structural coloration, while resonant scattering in core-shell geometry is also investigated for the first time. Interference from the shell and Mie scattering from the core region of the nanowires produce omnidirectional coloring within the very same structure, tuned by simply altering the overall diameter. Thermal size reduction can be regarded as a native wavelength-scalable nanowire based coloration technique since any hue of a particular color can be obtained by simply tuning the size reduction factor (Figure 1a).

Core-shell nanowires used here that exhibit double structural coloration are produced by a novel top-down size reduction process. This fabrication scheme has its own advantages compared to other bottom-up and top-down methods. Chemically synthesized nanowires (VLS) usually require postassembling and aligning of the nanostructures, and sometimes further processing such as polymer encapsulation for large area integration. ${ }^{15}$ Lithography, a widely used sophisticated technique, suffers from

Received: June 30, 2011

Revised: September 26, 2011

Published: October 18, 2011 

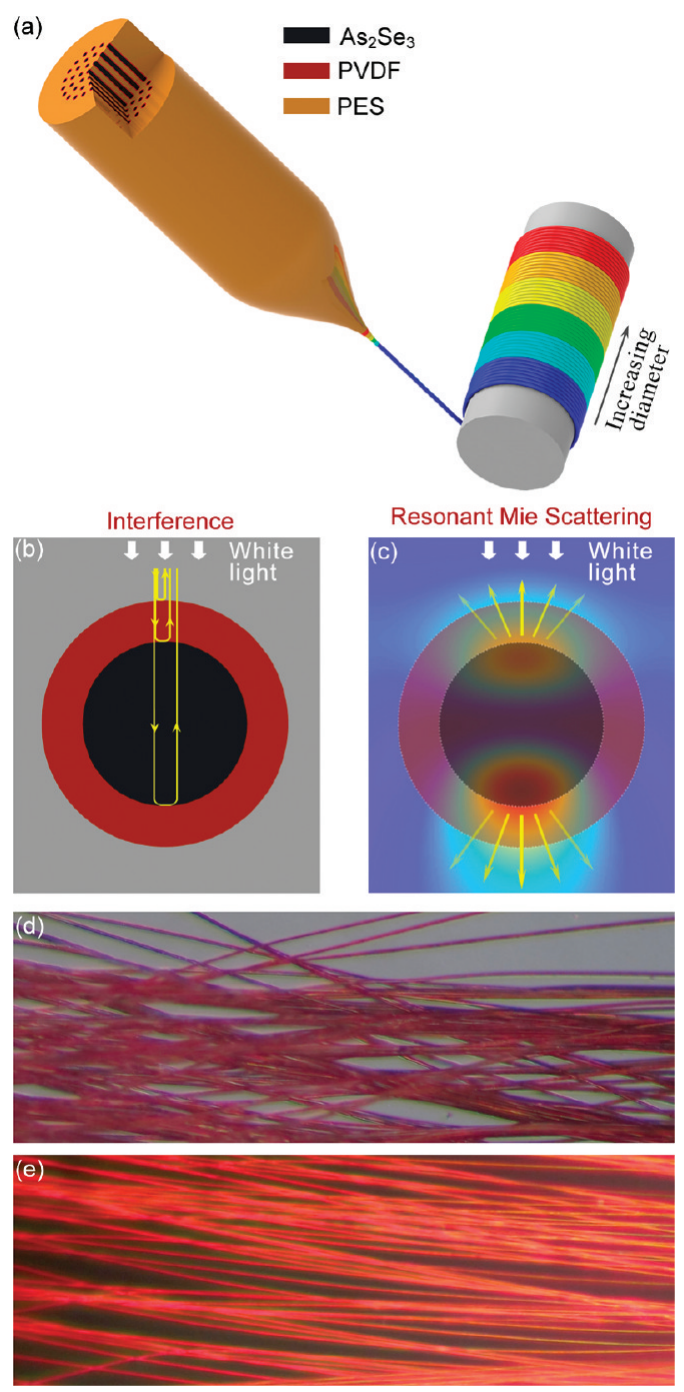

Figure 1. (a) A novel thermal size reduction technique is used to obtain indefinitely long, scalable core-shell nanowires. The structure consists of a high-dielectric semiconducting $\left(\mathrm{As}_{2} \mathrm{Se}_{3}\right.$, arsenic triselenide) core, piezoelectric polymer (PVDF, poly(vinylidene fluoride)) shell, and high-temperature thermoplastic (PES, polyethersulfones) cladding. The structures show native structural coloration based on their sizedependent properties. (b) Structural coloration based on nonresonant interference for 700-1200 nm diameter nanowires where coloration results from multiple reflections from core-shell interfaces. (c) Same core-shell structures, when scaled down to 30-200 nm diameter, also show resonant small particle scattering. Mass-produced, indefinitely long and large-area core-shell nanowire arrays colored by (d) thin-film interference and (e) resonant Mie scattering, respectively.

resolution and reproducibility, requires high cost equipment, and cannot be easily scaled up. Electrospinning is a high throughput method but it does not have flexibility in material set, or final geometry and size uniformity. The fabrication method recently developed by our group ensures that we obtain flexible polymer embedded, indefinitely long, ordered, uniform core-shell nanowires with precise control of physical and geometrical properties and chemical composition.

Nanowire fabrication by thermal drawing is based on size reduction of thermally compatible materials such as high temperature engineering polymers, low melting temperature chalcogen glasses, and low melting temperature metals. In order to reach nanodimensions, size reduction is iterated using the outcome of each preceding step in subsequent drawing steps. ${ }^{14}$ Core-shell nanowires are produced starting from a macroscopic $\mathrm{As}_{2} \mathrm{Se}_{3}$ glass rod with diameter of $4 \mathrm{~mm}$ and length of $15 \mathrm{~cm}$, wrapped in a poly(vinylidene fluoride) (PVDF) polymer sheet of $1 \mathrm{~mm}$ thickness, and consolidated in a polyethersulfone (PES) jacket, with total preform diameter of $25 \mathrm{~mm}$. PES is used for keeping whole structure together during thermal drawing, later to be removed in order to obtain free-standing core-shell nanowires, if required. Thermal drawing of the preform is carried out by controlling tension, temperature, feed-in, and drawing speed in a custom-built fiber tower optimized for nanowire production. After the first drawing step, we obtained $60 \mu \mathrm{m} \mathrm{As}{ }_{2} \mathrm{Se}_{3}$ microwires with a $15 \mu \mathrm{m}$ PVDF shell embedded in a PES fiber of $400 \mu \mathrm{m}$. Afterward, we cut 360 pieces of $15 \mathrm{~cm}$ long fibers obtained from the first step, stacked, embedded in a PES jacket, and consolidated them for a second drawing step. After the second thermal drawing, we obtained core-shell nanowires of $70-1800 \mathrm{~nm}$ by altering the reduction factor (Figure S1, Supporting Information). By repeating this procedure for the third time, we produced core-shell nanowires with diameters down to $10 \mathrm{~nm}$.

Chalcogen and polymer core-shell nanowires show novel coloration properties. In this study, we observe thin film interference based coloration from a core-shell nanowire structure, as well as Mie scattering from the core region (Figure $1 b-e$ ). The refractive index and the core diameter determine the scattered resonant modes in the vicinity of the nanowire, and thin film interference dominates for appropriate shell thicknesses. Both coloration mechanisms can be tuned over the whole visible spectrum by controlling the size reduction factor during nanowire fabrication. Absorption is apparently an undesirable property of the materials for structural color formation. PVDF has a refractive index of 1.41 and virtually no absorption in the visible range, rendering it a proper material for nanoshell coloration and an auxiliary in scattering. $\mathrm{As}_{2} \mathrm{Se}_{3}$ has a high refractive index varying from 3.36 to 2.95 and nonzero extinction coefficient below $700 \mathrm{~nm}$ (Figure S2, Supporting Information), but this does not prevent observing either coloration mechanism, with the compromise of slightly lower scattering efficiency. Scattering from individual core-shell structures obtained after second and third drawing steps is investigated under an inverted light microscope. The PES jacket is removed by dichloromethane (DCM) exposure to isolate the individual core-shell nanowires.

Upon bright field illumination, three core-shell nanowires with core diameter around $1 \mu \mathrm{m}(700-1200 \mathrm{~nm})$ and shell thicknesses of 170, 195, and $270 \mathrm{~nm}$ show lustrous primary colors occurring via thin film interference from the PVDF shells (Figure 2a). The PVDF shell layer and $\mathrm{As}_{2} \mathrm{Se}_{3}$ core region can be seen from the cross section of the core-shell nanowires (Figure $2 \mathrm{~b}$ ). The cross section is viewed after exposing it with ultramicrotomy. We used bright field microscopy and collected reflected light from the nanowires by coupling a spectrometer (Maya 2000 Pro VUV) to the microscope (Figure S3, Supporting Information). Efficient illumination spectrum of microscope lamp is limited by the range of $425-900 \mathrm{~nm}$; therefore, we fixed the spectra in this range. Measured spectra from three nanowires are shown in Figure 2c. Three distinct peaks, for the three coreshell nanowires, can be discerned at $465 \mathrm{~nm}$ (blue), $520 \mathrm{~nm}$ (green), and $740 \mathrm{~nm}$ (red), where the last peak is modulated by secondary interference from the core region. This modulation effect is suppressed for the first and second peaks due to the optical absorption of the core region. Finite difference time 
(a)

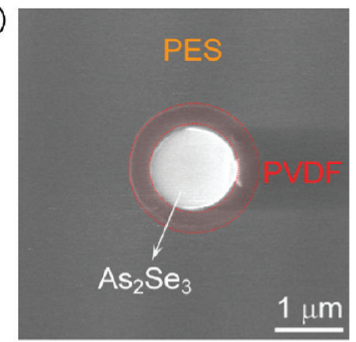

(b)

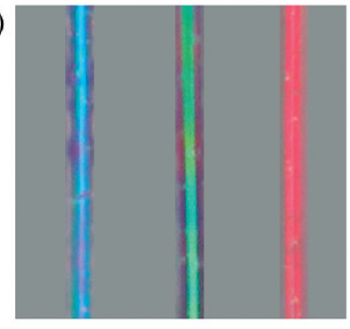

(c)

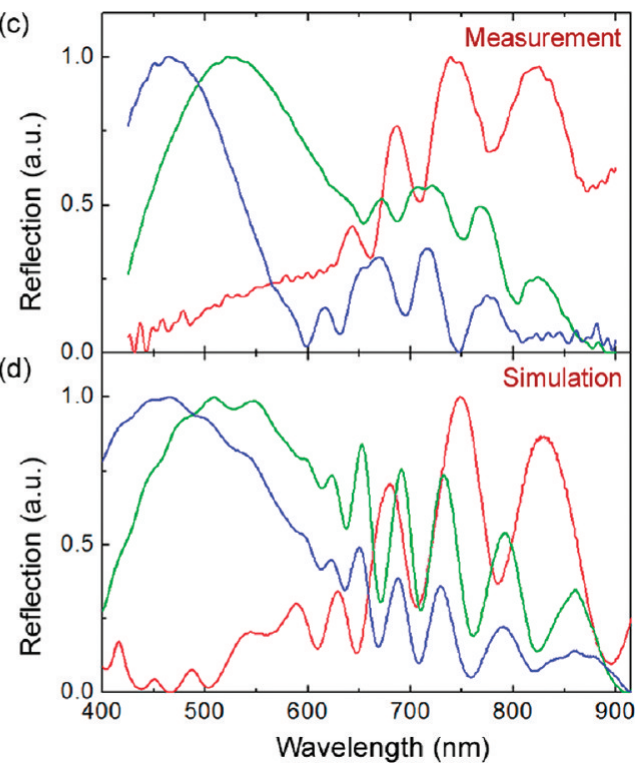

Figure 2. (a) SEM image of the polymer embedded $\mathrm{As}_{2} \mathrm{Se}_{3}$ (core)/PVDF (shell) microwire/nanoshell. (b) Size-dependent coloration from the shell thicknesses of the $170 \mathrm{~nm}$ (blue), $195 \mathrm{~nm}$ (green), and $270 \mathrm{~nm}$ (red). (c) Spectroscopic bright field reflection measurements from the three core-shell structures show size-dependent interference peaks at 465 (blue), 520 (green), and 740 (red). Modulation in the red spectra is due to secondary interference from the core region. (d) FDTD simulations with wavelength-dependent refractive index and extinction coefficients are in agreement with the measurements.

domain (FDTD) simulations are used to study the reflection from the core-shell nanowires, both in TM and in TE polarizations. Simulations are carried out using wavelength-dependent optical constants of $\mathrm{As}_{2} \mathrm{Se}_{3}$ and PVDF and using plane wave source. The reflection behavior is similar for both polarizations as expected; therefore, TM polarization is shown in the Figure $2 \mathrm{~d}$. The simulations and the measured data agree very well, manifesting both the primary interference from the shell and secondary interference from the core region. The position of maximum reflection from core-shell nanowires can be estimated from $m \lambda / n_{\text {shell }}=2 t_{\text {shell, }}$ where $m=1,2, \ldots$, is order of interference, $\lambda$ is the free space wavelength of incident light, $n_{\text {shell }}$ is the refractive index of shell layer, and $t_{\text {shell }}$ is the thickness of shell layer. For instance, the $270 \mathrm{~nm}$ thick PVDF shell results in a maximum reflection at $\lambda=761 \mathrm{~nm}$, which is very close to the measured and numerical FDTD results in panels $\mathrm{c}$ and $d$ of Figure 2.

As the core-shell nanowire diameters reduce to smaller diameters $(200-30 \mathrm{~nm})$, covering shell thicknesses decrease simultaneously due to the fabrication process and become extremely thin. The interference effect due to the covering shell layers is no longer observable for these core-shell nanowires, but it is now replaced by a new coloration mechanism originating from resonant Mie scattering. Incident electromagnetic waves having specific wavelength, $\lambda$, are trapped along the periphery of core-shell nanowires similar to whispering gallery modes in micrometer-scale resonators. ${ }^{16}$ The resonant field intensity is built up inside the nanowire and then the confined mode leaks due to the small size of nanowires compared to the wavelength of the light $(\lambda \gg d) .{ }^{17,13,9}$

We observed size-dependent coloring from nanowires having diameters smaller than $200 \mathrm{~nm}$. Primary colors were clearly observed for $175 \mathrm{~nm}$ (red), $42 \mathrm{~nm}$ (green), and $35 \mathrm{~nm}$ (blue) diameter core-shell nanowires after removal of PES (Figure 3a). Dark field illumination was used in order to increase the resolution of the images. The cross sections of the three core-shell nanowires are shown in the TEM image (Figure $3 \mathrm{~b}$ ). The shell layer is barely visible due to low contrast between PVDF and PES and smearing due to ultramicrotomy. We collected scattered light using the spectrometer. Measured spectra from three nanowires are shown in Figure 3c. Three resonant Mie scattering peaks were observed at $470 \mathrm{~nm}$ (blue), $530 \mathrm{~nm}$ (green), and $700 \mathrm{~nm}$ (red). FDTD simulations with wavelength-dependent refractive indices and extintion coefficients reproduce the measurement peaks confirming the resonant scattering effect from the core-shell nanowires (Figure 3d) assuming TM polarized light. Blue and green peaks are identified as $\mathrm{TM}_{01}$ and the red peak as $\mathrm{TM}_{11}$. The scattering efficiency of TE polarization is known to be smaller compared to the TM polarization. ${ }^{9,18} \mathrm{We}$ used the total field scattered field source to separate the computation domain into two distict regions, total and scattered fields, in the simulations. ${ }^{19}$ In the experiments, a shoulder peak observed to the left of the red peak is calculated to be the low efficiency $\mathrm{TE}_{11}$ resonant peak that does not effectively contribute to coloration. The increasing noise in the scattering intensity in Figure $3 c$ is due to the onset of $\mathrm{As}_{2} \mathrm{Se}_{3}$ absorption.

We can roughly predict the resonant scattering wavelength from $m \lambda / n_{\text {eff }}=\pi d$ where $m=1,2, \ldots, \lambda$ is the free space wavelength of incident light, $n_{\mathrm{eff}}$ is the effective refractive index, and $d$ is the diameter of the nanowire. For instance, the nanowire with $175 \mathrm{~nm}$ diameter exhibits a scattering peak at $695 \mathrm{~nm}$ as shown in Figure 3c. By using the above formula, we obtain $n_{\text {eff }}=$ 2.65 (for $m=2$ ), which is close to the refractive index of $\mathrm{As}_{2} \mathrm{Se}_{3}$ core (2.95) at this wavelength (see Figure S2, Supporting Information). Lower $n_{\text {eff }}$ is expected because the mode $\mathrm{TM}_{11}$ is not confined only at high-index core. The scattering efficiency and resonance wavelength can be directly calculated from Lorenz-Mie theory for unpolarized incident light ${ }^{18}$

$$
\mathrm{Q}_{\mathrm{sca}}(x)=\frac{1}{x_{n}} \sum_{-\infty}^{n=+\infty}\left(\left|a_{n}\right|^{2}+\left|b_{n}\right|^{2}\right)
$$

where $x=k d$ and the scattering coefficients $a_{n}$ and $b_{n}$ are obtained by solving Maxwell's equations with boundary conditions at 
(a)

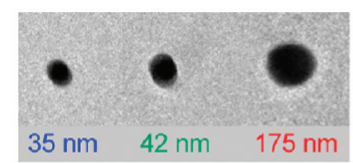

(b)

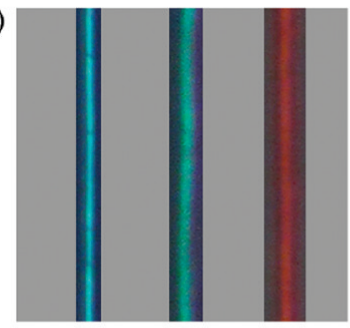

(c)

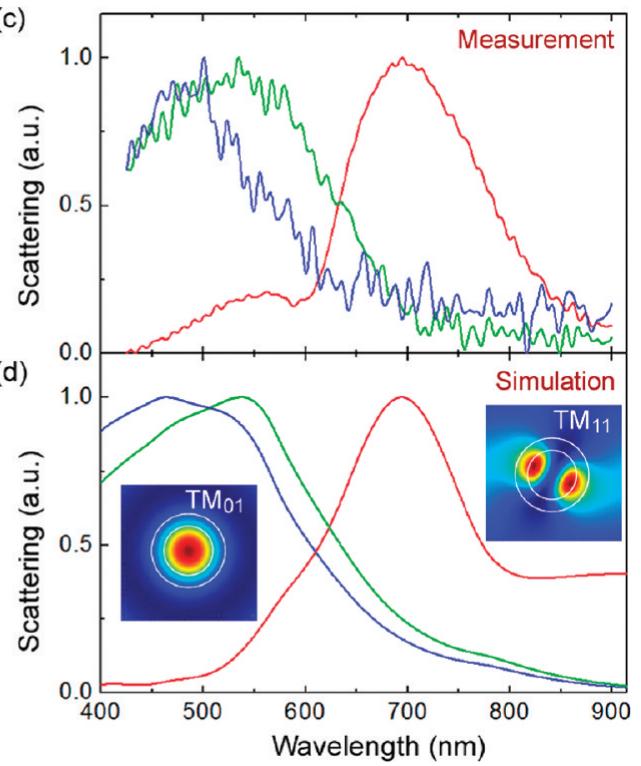

Figure 3. (a) TEM images of polymer embedded $\mathrm{As}_{2} \mathrm{Se}_{3} / \mathrm{PVDF}$ core-shell nanowires. (b) Size-dependent Mie scattering from $35 \mathrm{~nm}$ (blue), $42 \mathrm{~nm}$ (green), and $175 \mathrm{~nm}$ (red) core-shell nanowires. (c) Spectroscopic dark field scattering measurements from these three core-shell nanowires show size-dependent resonant peaks at 470 (blue), 530 (green), and 700 (red). (d) Numerical FDTD calculations of scattering from these nanowires agree well with the measurements. Inset: Resonant modes are identified using FDTD simulations. In the calculations, we used wavelength-dependent refractive indices and extinction coefficients obtained from spectroscopic ellipsometry measurements.

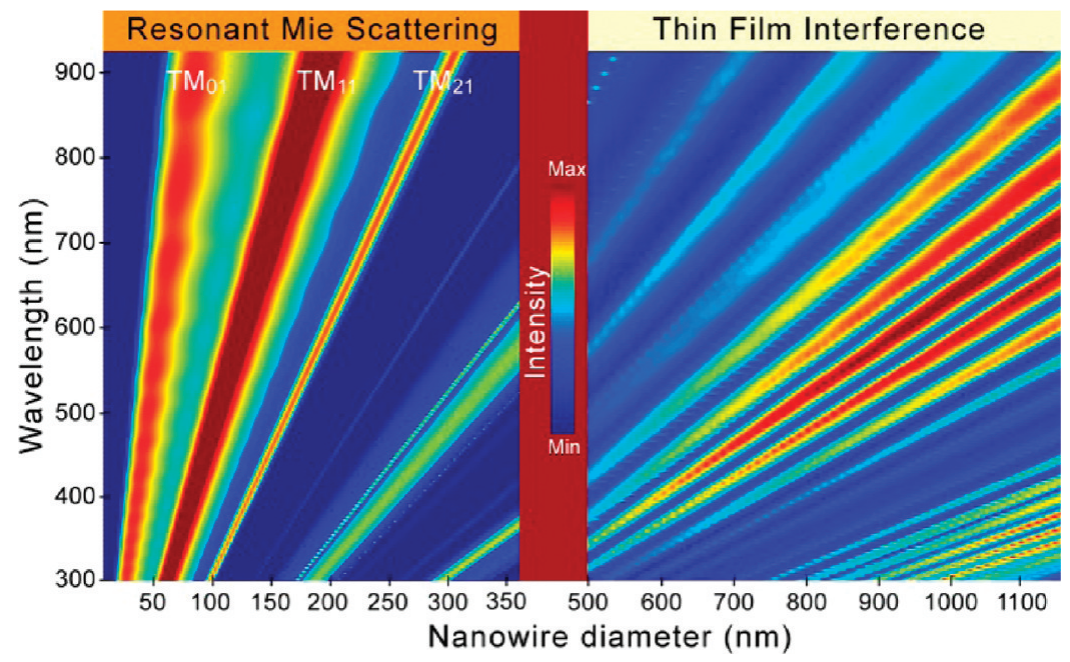

Figure 4. Two complementary structural coloring schemes, small particle scattering, and thin film interference are demonstrated from FDTD simulations of size scalable $\mathrm{As}_{2} \mathrm{Se}_{3}$ /PVDF core-shell nanowires using TM polarized light. Resonant Mie scattering is dominant for nanowire diameters from 30 to $200 \mathrm{~nm}$. Resonant scattering peaks are identified as TM modes. Thin-film interference-based color formation is due to shell layer spans from 600 to $1200 \mathrm{~nm}$ diameter range. A secondary high-frequency interference for larger nanowires does not compromise coloration.

interfaces. The results obtained from the analytical calculations (not shown) agree well with measurements and simulations.

The core-shell nanowire structure features a double coloring scheme that is comprised of interference based coloring from the shell and scattering based coloring from the core. With the size reduction factor set during fabrication, core-shell nanowires can be produced that cover the full visible spectrum (Figure 4). With FDTD simulations, both scattering efficiency and reflection characteristics of core-shell nanowires are investigated in the visible spectrum (300-900 $\mathrm{nm}$ ) for varying nanowire diameters $(20-$ $1200 \mathrm{~nm}$ ). Simulations are performed in the TM polarization using a constant refractive index of 3.25 for $\mathrm{As}_{2} \mathrm{Se}_{3}$ core and 1.41 for PVDF shell. For core-shell nanowire diameters below $300 \mathrm{~nm}$, where the PVDF shell is far thinner to support thin film interference in the visible range, Mie scattering from the core dominates coloration. The core diameter determines the structure and the number of whispering gallery type modes resonating inside the nanowire (Figure S5, Supporting Information). The resonance conditions for the Mie scattering case are identified as $\mathrm{TM}_{01}, \mathrm{TM}_{11}$, and $\mathrm{TM}_{21}$. A single resonant mode is observed for dimensions smaller than $50 \mathrm{~nm}$, and additional modes appear for increasing diameter of nanostructures. For larger dimensions, on the other hand, interference effects become a major coloration mechanism. The interference pattern consists of a slowly varying 
shell region interference modulated by a rapid oscillation from the thicker core region. The modulation does not affect coloration from the shell region. Material absorption manifests itself by suppressing rapidly varying modulations especially for smaller wavelengths in the interference pattern. For Mie scattering, material absorption results in the omission of higher order modes and decreased scattering efficiency.

The nanostructures obtained by the new fabrication method are unique in their coloration features. They exhibit and are suited for both interference based and small particle scattering based coloring. ${ }^{9}$ These core-shell nanowires have two characteristic radial size scales that give rise to respective coloration mechanisms. More importantly, simply by altering drawing conditions, i.e., changing the reduction factor, any hue from the visible spectrum can be effectively obtained. High refractive index chalcogenide glasses enhance interference effects and increase scattering efficiency at nanodimensions. However, they have finite extinction coefficients for lower visible spectrum, slightly compromising the scattering efficiency of the structure. PVDF, with a relatively low refractive index, causes high Fresnel reflection from the polymer/chalcogenide interface. Iterative thermal size reduction technique results in indefinitely long coreshell nanowire, nanowires, or nanotubes having diameter uniformity at macroscopic length scales which results in uniform coloring of large area nanowires. The PVDF shell layer around nanowires significantly reduces the cross-coupling (which can change colors of nanowires at interaction regions) between nanowires which is critical for a dense nanowire array. Moreover by changing the thickness of a shell layer one can tune the coupling strength between nanowires.

In conclusion, we demonstrated two different mechanisms for structural coloration in large scale core-shell nanowires. One of these mechanisms, nanoshell coloration, which is intrinsic to the core-shell geometry, is proposed as a new coloration method. The nanostructures are produced with a novel high-throughput fabrication technique for mass production and suitable application on flexible surfaces. Polymer fiber embedded one-dimensional nanostructures can be used singly or collectively, using array based coloration or photonic crystal based coloration, as iridescent or solid color centers. In addition to textile and decoration applications, nanostructures can be used as functional modules as solar cell concentrators, as stimuli responsive polymers, and in biological imaging, providing unique applications. The two-dimensional lattice of nanowires may exhibit negative index of refraction at optical domain, ${ }^{20}$ and one-dimensional coupled core-shell nanowire arrays can be used to generate slow light. $^{21}$

\section{ASSOCIATED CONTENT}

S Supporting Information. Core-shell nanowire production scheme, optical properties of chalcogenide glass $\left(\mathrm{As}_{2} \mathrm{Se}_{3}\right)$ and piezoelectric polymer (PVDF), experimental setup for reflection and scattering measurements, reflection from structures associated with cylindrical core-shell nanowires, Mie scattering analysis, and mode profiles. This material is available free of charge via the Internet at http://pubs.acs.org.

\section{AUTHOR INFORMATION}

\section{Corresponding Author}

*E-mail: bayindir@nano.org.tr.

\section{ACKNOWLEDGMENT}

We thank Mehmet Kanik for his help in fiber drawing, Ekin O. Ozgur for SEM images, and Dr. Hakan Deniz for TEM micrographs. This work was partially supported by the Ministry of Development and TUBITAK under the Project No. 110M412 and 106G090. M.B. acknowledges support from the Turkish Academy of Sciences Distinguished Young Scientist Award (TUBA GEBIP).

\section{REFERENCES}

(1) Kinoshita, S.; Yoshioka, S.; Miyazaki, J. Rep. Prog. Phys. 2008, 71, 076401.

(2) Rahman, F.; Johnson, N. P. Opt. Photonics News 2008, 19 (2), 24-29.

(3) Hart, S. D.; Maskaly, G. R.; Temelkuran, B.; Prideaux, P. H.; Joannopoulos, J. D.; Fink, Y. Science 2002, 296, 510-513.

(4) Abouraddy, A. F.; Bayindir, M.; Benoit, G.; Hart, S. D.; Kuriki, K.; Orf, N.; Shapira, O.; Sorin, F.; Temelkuran, B.; Fink, Y. Nat. Mater. 2007, 6, 336-347.

(5) Gurel, K.; Kaplan, B.; Guner, H.; Bayindir, M.; Dana, A. Appl. Phys. Lett. 2009, 94, 233102.

(6) Kinoshita, S.; Yoshioka, S. Chem. Phys. Chem. 2005, 6, 1442-1459.

(7) Braun, P. V. Nature 2011, 472, 423-424.

(8) Finlayson, C. E.; Spahn, P.; Snoswell, D. R. E.; Yates, G.; Kontogeorgos, A.; Haines, A. I.; Hellmann, G. P.; Baumberg, J. J. Adv. Mater. 2011, 23, 1540-1544.

(9) Cao, L.; Fan, P.; Barnard, E. S.; Brown, A. M.; Brongersma, M. L. Nano Lett. 2010, 10, 2649-2654.

(10) Seo, K.; Wober, M.; Steinvurzel, P.; Schonbrun, E.; Dan, Y.; Ellenbogen, T.; Crozier, K. B. Nano Lett. 2011, 11, 1851-1856.

(11) Xiao, Y.; Meng, C.; Wang, P.; Ye, Y.; Yu, H.; Wang, S.; Gu, F.; Dai, L.; Tong, L. Nano Lett. 2011, 11, 1122-1126.

(12) Cao, L; Fan, P.; Vasudev, A. P.; White, J. S.; Yu, Z.; Cai, W.; Schuller, J. A.; Fan, S.; Brongersma, M. L. Nano Lett. 2010, 10, 439-445.

(13) Cao, L.; White, J. S.; Park, J. S.; Schuller, J. A.; Clemens, B. M.; Brongersma, M. L. Nat. Mater. 2009, 8, 643-647.

(14) Yaman, M.; Khudiyev, T.; Ozgur, E.; Kanik, M.; Aktas, O.; Ozgur, E. O.; Deniz, H.; Korkut, E.; Bayindir, M. Nat. Mater. 2011, 10, 494-501.

(15) Huang, Y.; Duan, X. F.; Wei, Q. Q.; Lieber, C. M. Science 2001, 291, 630-633.

(16) Vahala, K. J. Nature 2003, 424, 839-846.

(17) Wang, J.; Gudiksen, M. S.; Duan, X. F.; Cui, Y.; Lieber, C. M. Science 2001, 293, 1455-1457.

(18) Bohren, C. F.; Huffman, D. R. Absorption and Scattering of Light by Small Particles; John Wiley \& Sons Inc.: New York, 1998.

(19) Detailed information can be obtained from http://docs.lumerical. com/en/fdtd/user_guide_tfsf_sources.html

(20) Vynck, K.; Felbacq, $\bar{D}$.; Centeno, E.; Cabuz, A. I.; Cassagne, D.; Guizal, B. Phys. Rev. Lett. 2009, 102, 133901.

(21) Bayindir, M.; Temelkuran, B.; Ozbay, E. Phys. Rev. Lett. 2000, $84,2140-2143$. 